\title{
Téoros
}

Revue de recherche en tourisme

\section{Les congrès et les retombées économiques}

\section{Alain Dubuc}

Volume 4, numéro 3, novembre 1985

L'essor de l'industrie des congrès

URI : https://id.erudit.org/iderudit/1080795ar

DOI : https://doi.org/10.7202/1080795ar

Aller au sommaire du numéro

Éditeur(s)

Université du Québec à Montréal

ISSN

0712-8657 (imprimé)

1923-2705 (numérique)

Découvrir la revue

Citer cet article

Dubuc, A. (1985). Les congrès et les retombées économiques. Téoros, 4(3),

34-35. https://doi.org/10.7202/1080795ar d'utilisation que vous pouvez consulter en ligne.

https://apropos.erudit.org/fr/usagers/politique-dutilisation/ 


\title{
Les congrès \\ et les retombées économiques
}

\author{
par Alain Dubuc*
}

Neuf fois sur dix, quand un organisme, une compagnie, un ministre ou un promoteur utilise le concept de retombées économiques pour justifier ses actions, il faut se méfier.

En effet, c'est sans doute l'outil économique le plus galvaudé, le plus vidé de son sens. Dans la plupart des cas, il faudrait carrément le bannir de son vocabulaire.

II $\mathrm{y}$ a heureusement quelques rares exceptions. L'une d'entre elles, c'est l'industrie des congrès dont profite largement la région montréalaise. Lorsqu'un congrès d'envergure internationale se tient à Montréal, on peut effectivement parler de retombées.

\section{Un concept qui a mal tourné}

Pourtant, quand on s'en sert à bon escient, le calcul des retombées économiques est une technique fort pratique. A l'origine, c'est un élément important de l'analyse bénéfice-coût, un outil utilisé par les économistes pour effectuer des choix entre divers projets. Par exemple, l'Etat a 200 millions \$a injecter dans un secteur de pointe: faut-il l'investir dans une usine de montage d'autos ou dans une usine d'hélicoptères? Faut-il construire un port à Sept-lles ou à Baie Comeau?

En mesurant les retombées de chaque alternative, il est possible de déterminer laquelle est la plus rentable sur le plan économique, en terme d'emplois, d'investissements, de revenus, de modification de la structure économique.

Dans le cadre de ce genre de démarche, on utilise d'habitude des multiplicateurs, en se basant sur le raisonnement suivant: un dollar investi quelque part aura un impact final supérieur à un dollar. Par exemple, si on investit 20 millions $\$$ dans un centre de ski, ce 20 millions $\$$ créera des emplois; les salaires payés permettront de consommer des biens et créeront donc d'autres emplois, et ainsi de suite.

\footnotetext{
"Alain Dubuc est chroniqueur économique au journal La Prasse.
}

Les multiplicateurs ne sont cependant pas les mêmes selon le secteur ou les formes de dépenses. Par exemple, un dollar investi dans une usine de montage automobile aura un impact autrement plus profond que le mềme dollar consacré à l'achat d'un stand de patates frites.

\section{La multiplication}

Pendant longtemps, cette méthode est restée bien à l'abri dans les officines des économistes jusqu'à ce que les gouvernements décident de s'en servir. Ce fut le debut de la fin.

Un ministre qui annonçait un investissement de 100 millions $\$$ à rapidement compris qu'il était beaucoup plus rentable sur le plan politique d'affirmer froidement que l'impact de sa décision atteindrait 250 millions \$. grâce à la magie des multiplicateurs. On pouvait, de la même façon, en tenant compte des emplois indirects, annoncer deux fois plus d'emplois créés.
La même méthode a longtemps encouragé les gouvernements a affirmer froidement que certains de leurs projets ne coutteraient rien à l'Etat en bout de ligne, parce cue les retombées seraient telles que les rentrées d'impôt financeraient la dépense. On sait maintenant ce que cela a fait au déficit!

\section{L'arrivée des promoteurs}

La méthode n'est pas tombée dans l'areille de sourds. Au bout d'un certain nombre d'années, surtout à partir de la fin des annees soixante-dix, les promoteurs, les demandeurs de subventions se sont mis à utiliser ce truc pour présenter leurs projets sous un jour plus favorable.

Cette methode, utilisee hors contexte, comporte trois défauts graves. Le premier est l'utilisation abusive des multiplicateurs. N'importe qui peut maintenant multiplier l'impact de son projet par 2,25 ou 2,5. Ca ne veut strictement rien dire.

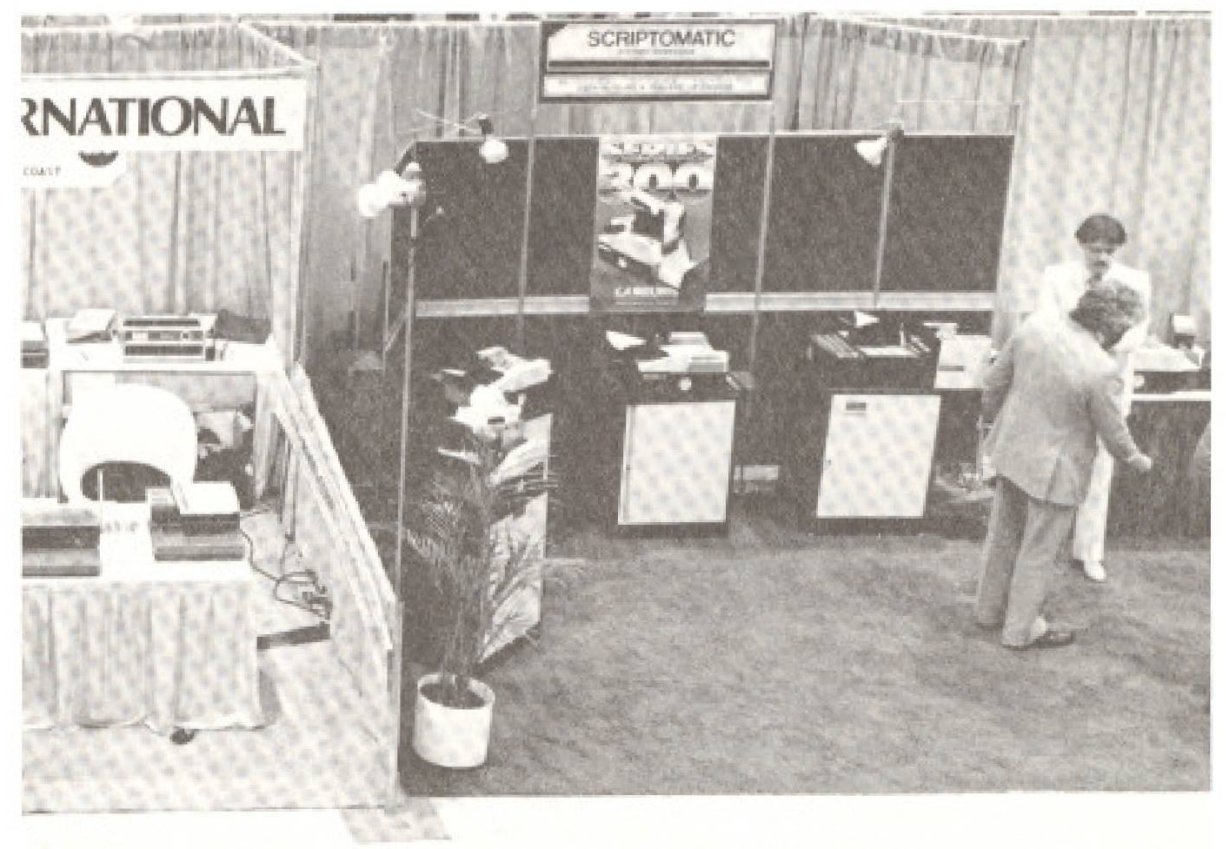

Un congrès: $c^{\prime e s t ~ d e ~ I ’ a r g e n t ~ f r a i s ~ i n j e c t e ́ ~ d a n s ~ l e ~ c i r c u i t ~ e ́ c o n o m i q u e ~ q u e ́ b e ̀ c o i s . ~}$ 
Avec ce genre de raisonnement, n'impoprte quelle activité économique peut ètre multipliée. Quand vous prenez un scotch dans un bar, qui vous coüte $3,50 \$$, cette approche vous permet tout à fait d'en déduire que vous venez de permettre une injection finale de 8,75 \$ dans l'économie. Pourquoi ne pas multiplier tout de suite le PNB par 2,5?

Le second défaut de tous ces savants calculs, c'est que les retombées d'un projet ne sont souvent possibles qu'en diminuant I'activité économique ailleurs. Par exemple, la construction d'un centre d'achat à Montréal n'aura les retombées que l'on invoque que si ce nouveau complexe commercial réussit à s'accaparer la clientèle de magasins déjầ existants. L'économie dans son ensemble n'en profite pas nécessairement.

Le même raisonnement vaut pour plusieurs événements touristiques. Le Festival western de St-Tite, par exemple, permet de générer un bon chiffre d'affaires parce qu'il attire des amateurs d'un peu partout. Ce sont des retombées pour St-Tite et la Mauricie, mais c'est de l'argent qui n'a pas été dépensé ailleurs.

Enfin, le concept de retombées n'a de sens que si on introduit des critères qualitatifs. Une retombée économique n'est pas forcément souhaitable. Un exemple suffit à le montrer. Un accident d'auto est riche en retombées économiques: touage, garagistes, ambulances etc. II le sera encore plus si I'automobiliste abime trois autres véhicules et démolit une vitrine.

\section{Les congrès, une catégorie à part}

On voít donc les limites très sérieuses de cet outil. Dans le cas des congrès, c'est toutefois différent, si l'événement permet la tenue d'activités qui autrement n'auraient pas eu lieu ici.

Un congrès international est un ajout net à la vie économique. Si Montréal ne l'avait pas obtenu, ce serait sans doute une ville américaine qui aurait été gagnante. II permet donc au Canada d'obtenir des devises étrangères, toujours bienvenues, et à Montréal de profiter de dépenses qui auraient pu être faites dans un autre pays. C'est donc une injection de capitaux frais. Pour employer une expression à la mode, c'est de l'argent frais injecté dans le circuit économique québécois.

II faut toutefois apporter quelques nuances. Un congrès génère des revenus, mais il comporte aussi des coûts, dont il faut tenir compte. Un événement qui remplit les hótels de Montréal prive l'industrie touristique d'autres revenus, les visiteurs pour qui il n'y a plus de place dans la métropole. Le gigantisme de certains événements comporte enfin des dépenses supplémen- taires, liées à la congestion, au besoins d'infrastructures et de personnel additionnel.

D'autres événements, que l'on associe souvent aux congrès, ne permettent pas un calcul précis de retombées. C'est par exemple le cas des Floralies, des expositions comme Ramsès ou Picasso où il est difficile de départager les revenus provenant des étrangers et surtout où il est impossible d'affirmer que c'est l'événement luimême qui a attiré des visiteurs áa Montréal.
Mais, dans l'ensemble, le calcul des retombées économiques reste un outil très par= tiel parce qu'il ne permet pas de mesurer les impacts qui sont les plus importants. Un congrès, c'est beaucoup plus que les sommes consacrées à l'hébergement et à la restauration. C'est une façon de faire de Montréal une ville de premier plan, de la faire découvrir à des gens qui peut-être ne l'auraient jamais découverte; à long terme, les congrès ont ainsi impact touristique, économique, culturel et politique qu'aucun ordinateur ne peut chiffrer.

\section{Suite de la page 33 \\ Le choix d'un site de congrès}

\section{L'expérience de l'ordre des architectes du Québec}

quelques jours les avantages de l'infrastructure montréalaise en matière de tourisme de congrès constitue un élément très positif dans le choix éventuel de Montréal comme site du Congrès UIA-1990 et ceci malgré les difficultés inhérentes d'organisation, plus particulièrement au niveau du financement.

Dans un article paru dans le cahier spéciale de la Presse en date du 14 septembre 1985 , le président directeur général du Palais des Congrẻs, monsieur Yves Michaud, fait état du fait que "solliciter une association internationale pour que son congres se tienne à Montréal peut coüter très cher... le coût de sollicitation, dépendant des conditions dans lesquelles elle se déroule, peut s'élever entre $100 \mathrm{~s}$ et 100000 \$. Dans certains cas, nous devons, à titre d'argument de vente, produire un document audiovisuel elaboré et coûteux. Ce fut le cas lors de la sollicitation du congrès de l Union internationale des architectes que nous avons obtenu au Caire. .... c'est un peu beaucoup grace a la qualite de notre document audio-visuel que nous avons coiffe nos concurrents au fill d'arrivée." Le financement (ou le pré-financement) constitue donc la pierre d'achoppement principale dans la présentation d'une candidature de sollicitation d'un congrès international. Dans le cas qui nous intéresse, cette difficulté fut surmontée grâce à des arrangements de toute dernière minute qui souvent durent mettre carrément de côté́ les directives ou les normes prévues à cet effet.

II n'est pas du ressort de cet article d'examiner ce point davantage, mais c'est certainement là un sujet intéressant et fondamental pour la sollicitation de congrès internationaux.

\section{Le Caire, janvier 1985 - XVe Congrès mondial de I'UIA}

Une fois la phase préparatoire complétée, il restait donc à la délégation choisie de présenter le dossier de candidature dans le cadre du XVe Congrès international de I'UIA au Caire en janvier 1985. La délégation canadienne se composait de représentants de I'OAQ, de l'IRAC et du Palais des Congrès; sans rapporter en détail toutes les péripéties de cette aventure plus que fascinante à plusieurs égards (encore du matériel pour une autre publication), I'on ne peut passer sous silence le mode de sélection des sites de congrès tel qu'appliqué à I'UIA: c'est l'assemblée générale qui décide et non l'exécutif ou le conseil; l'assemblée générale est composée de représentants des 90 pays membres au prorata du nombre d'architectes sur leur territoire; ainsi, à titre d'exemple, mentionnons que l'URSS a 7 délégués-votants, le Canada 5 et I'Ouganda 1. Ceci oriente évidemment toute la stratégie autant durant la phase préparatoire qu'au moment de la présentation comme telle de la candidature.

Le résultat de tous ces efforts concertés est évidemment que Monsréal sera en juin 1990 la ville-hôte du XVIle Congrès mondial de I'UIA qui amènera dans nos murs un minimum de 6000 participants (architectes, accompagnateurs, étudiants, etc.); ce dossier de sollicitation fut également à maints égards une expérience-pilote dont il faudra tenir compte dans les dossiers analogues à venir: un "debriefing" systématique de toutes les phases de l'opération a été fait et l'information qui en est résultée est déjà mise à contribution dans d'autres dossiers. 